\title{
Towards the Production of Second Generation Ethanol from Sugarcane Bagasse in Brazil
}

\author{
T.P. Basso, T.O. Basso, C.R. Gallo and L.C. Basso
}

Additional information is available at the end of the chapter

http://dx.doi.org/10.5772/54179

\section{Introduction}

Brazil and the United States produce ethanol mainly from sugarcane and starch from corn and other grains, respectively, but neither resource are sufficient to make a major impact on world petroleum usage. The so-called first generation (1G) biofuel industry appears unsustainable in view of the potential stress that their production places on food commodities. On the other hand, second generation $(2 \mathrm{G})$ biofuels produced from cheaper and abundant plant biomass residues, has been viewed as one plausible solution to this problem [1]. Cellulose and hemicellulose fractions from lignocellulosic residues make up more than two-thirds of the typical biomass composition and their conversion into ethanol (or other chemicals) by an economical, environmental and feasible fermentation process would be possible due to the increasing power of modern biotechnology and (bio)-process engineering [2].

Brazil is the major sugar cane producer worldwide (ca. 600 million ton per year). After sugarcane milling for sucrose extraction, a lignocellulosic residue (sugarcane bagasse) is available at a proportion of ca. $125 \mathrm{~kg}$ of dried bagasse per ton of processed sugarcane. Therefore, sugarcane bagasse is a suitable feedstock for second generation ethanol coupled to the first generation plants already in operation, minimizing logistic and energetic costs.

State-owned energy group Petrobras is one of the Brazilian groups leading the development of second generation technologies, estimating that commercial production could begin by 2015. Other organizations making significant contributions to next generation biofuels in Brazil include the Brazilian Sugarcane Technology Centre (CTC), operating a pilot plant for the production of ethanol from bagasse in Piracicaba (Sao Paulo) [3]. Recently, GraalBio (Grupo Brasileiro Graal) has stated publically that will start production of bioethanol from sugarcane bagasse in one plant located at the Northeast region of the country. 
Pretreatment, hydrolysis and fermentation can be performed by various approaches. According to a CTC protocol, the process of manufacturing ethanol from bagasse is divided into the following steps. First, the bagasse is pretreated via steam explosion (with or without a mild acid condition) to increase the enzyme accessibility to the cellulose and promoting the hemicellulose hydrolysis with a pentose stream. The lignin and cellulose solid fraction is subjected to cellulose hydrolysis, generating a hexose-rich stream (mainly composed of glucose, manose and galactose). The final solid residue (lignin and the remaining recalcitrant cellulose) is used for heating and steam generation. The hexose fraction is mixed with $1 \mathrm{G}$ cane molasses (as a source of minerals, vitamins and aminoacids) and fermented by regular Saccharomyces cerevisiae industrial strains (not genetically modified) using the same fermentation and distillation facilities of the Brazilian ethanol plants. The pentose fraction will be used as substrate for other biotechnological purposes, including ethanol fermentation.

Researchers are focusing on cutting the cost of the enzymes and the pretreatment process, as well as reducing energy input. Production of ethanol from sugarcane bagasse will have to compete with the use of bagasse for electricity cogeneration. Depending on the efficiency of the cogeneration plant, about half of the bagasse is required to produce captive energy in the form of steam and energy at the sugar and ethanol facility. It is estimated that the surplus bagasse could increase the Brazilian ethanol production by roughly $50 \%$ [3].

\section{Lignocellulosic residues}

Lignocellulosic residues are composed by three main components: cellulose, hemicellulose and lignin. Cellulose and hemicellulose are polyssacharydes composed by units of sugar molecules [4]. Sugarcane bagasse is composed of around 50\% cellulose, 25\% hemicellulose and 25\% lignin. It has been proposed that because of its low ash content (around 2-3\%), this product offers numerous advantages in comparison to other crop residues (such as rice straw and wheat straw) when used for bio-processessing purposes. Additionally, in comparison to other agricultural residues, bagasse is considered a richer solar energy reservoir due to its higher yields on mass/area of cultivation (about $80 \mathrm{t} / \mathrm{ha}$ in comparison to about 1, 2, and $20 \mathrm{t} /$ ha for wheat, other grasses and trees, respectively) [5].

\subsection{Cellulose}

Cellulose is composed of microfibrils formed by glucose molecules linked by $\beta-1,4$, being each glucose molecule reversed in relation to each other. The union of microfibrils form a linear and semicrystalline structure. The linearity of the structure enables a strong bond between the microfibrils. The crystallinity confers resistance to hydrolysis due to absence of water in the structure and the strong bond between the glucose chains prevents hydrolases act on the links $\beta-1,4[1]$.

\subsection{Hemicellulose}

Hemicellulose is a polysaccharide made of polymers formed by units of xylose, arabinose, galactose, manose and other sugars, that present crosslinking with glycans. Hemicellulose 
can bind to cellulose microfibrils by hydrogen bonds, forming a protection that prevents the contact between microfibrils to each other and yielding a cohesive network. Xyloglucan is the major hemicellulose in many primary cell walls. Nevertheless, in secondary cell wall, which predominate in the plant biomass, the hemicelluloses are typically more xylans and arabino-xylans. Typically, hemicellulose comprises between 20 to $50 \%$ of the lignocellulose polysaccharides, and therefore contributes significantly to the production of liquid biofuels [1]. Sugarcane bagasse contain approximately $25-30 \%$ hemicelluloses $[13,14]$.

\subsection{Lignin}

Lignin is a phenolic polymer made of phenylpropanoid units [13], which has the function to seal the secondary cell wall of plants. Besides providing waterproofing and mechanical reinforcement to the cell wall, lignin forms a formidable barrier to microbial digestion. Lignin is undoubtedly the most important feature underlying plant biomass architecture. Sugarcane bagasse and leaves contain approximately $18-20 \%$ lignin [13]. The phenolic structure of this polymer confers a material that is highly resistant to enzymatic digestion. Its disruption represents the main target of pretreatments before enzymatic hydrolysis.

\section{Pretreatment}

The pretreatment process is performed in order to separate the carbohydrate fraction of bagasse and other residues from the lignin matrix. Another function is to minimize chemical destruction of the monomeric sugars [6]. During pretreatment the inner surface area of substrate particles is enlarged by partial solubilization of hemicellulose and lignin.. This is achieved by various physical and/or chemical methods [5]. However, it has been generally accepted that acid pretreatment is the method of choice in several model processes [7]. One of the most cost-effective pretreatments is the use of diluted acid (usually between 0.5 and $3 \%$ sulphuric acid) at moderate temperatures. Albeit lignin is not removed by this process, its disruption renders a significant increase in sugar yield when compared to other processes [1]. Regarding sugarcane bagasse several attempts have been made to optimize the release of the carbohydrate fraction from the lignin matrix, including dilute acid pretreatment, steam explosion, liquid hot water, alkali, peracetic acid and also the so called ammonia fiber expansion (AFEX).

\section{Hydrolysis}

Cellulose and hemicellulose fractions released from pretreatment has to be converted into glucose and other monomeric sugars. This can be achieved by both chemically- or enzymatically- oriented hydrolysis [7, 8].

\subsection{Chemical hydrolysis}

Whitin chemical hydrolysis, acid hydrolysis is the most used and it can be performed with several types of acids, including sulphurous, sulphuric, hydrochloric, hydrofluoric, 
phosphoric, nitric and formic acid. While processes involving concentrated acids are usually operated at low temperatures, the large amount of acids required may result in problems associated with equipment corrosion and energy demand for acid recovery. These processes typically involve the use of $60-90 \%$ concentrated sulfuric acid. The primary advantage of the concentrated acid process in realtion to diluted acid hydrolysis is the high sugar recovery efficiency, which can be on the order of $90 \%$ for both xylose and glucose. Concentrated acid hydrolysis disrupts the hydrogen bonds between cellulose chains, converting it into a completely amorphous state [8].

On the other hand, during dilute acid hydrolysis temperatures of $200-240^{\circ} \mathrm{C}$ at $1.5 \%$ acid concentrations are required to hydrolyze the crystalline cellulose. Besides that, pressures of 15 psi to 75 psi, and reaction time in the range of $30 \mathrm{~min}$ to $2 \mathrm{~h}$ are employed. During this conditions degradation of monomeric sugars into toxic compounds and other non-desired products are inevitable [9]. The main advantage of dilute acid hydrolysis in comparison to concentrated acid hydrolysis is the relatively low acid consumption. However, high temperatures required to achieve acceptable rates of conversion of cellulose to glucose results in equipment corrosion [4].

\subsection{Enzymatic hydrolysis}

Differently from acid hydrolysis, biodegradation of sugarcane bagasse by cellulolytic enzymes can be performed at much lower temperatures (around $50^{\circ} \mathrm{C}$ or even lower). Moreover conversion of cellulose and hemicellulose polymers into their constituent sugars is very specific and toxic degradation products are unlikely to be formed. However, a pretreatment step is required for enzymatic hydrolysis, since the native cellulose structure is well protected by the matrix compound of hemicellulose and lignin [4].

Cellulase is the general term for the enzymatic complex able to degrade cellulose into glucose molecules. The mechanism action accepted for hydrolysis of cellulose are based on synergistic activity between endoglucanase (EC 3.2.1.4), exoglucanase (or cellobiohydrolase (EC 3.2.1.91)), and $\beta$-glucosidase (EC 3.2.1.21). The first enzyme cleaves the bounds $\beta-1,4-$ glucosidic of cellulose chains to produce shorter cello-dextrins. Exoglucanase release cellobiose or glucose from cellulose and cello-dextrin chains and, finally $\beta$-glucosidases hydrolyze cellobiose to glucose. The intramolecular $\beta$-1,4-glucosidic linkages are cleaved by endoglucanases randomly. Endoglucanases and exoglucanases have different modes of action. While endoglucanase hydrolyze intramolecular cleavages, exoglucanases hydrolyze long chains from the ends. More specifically, exoglucanases or cellobiohydrolases have action on the reducing ( $\mathrm{CBH}$ I) and non-reducing ( $\mathrm{CBH}$ II) cellulose chain ends to liberate glucose and cellobiose. These enzymes acts on insoluble cellulose, then their activity are often measured using microcrystalline cellulose. Lastly, $\beta$-glucosidases or cellobioase hydrolyze cellobiose to glucose. They are important to the process of hydrolysis because they removed cellobiose to the aqueous phase that is an inhibitor to the action of endoglucanases and exoglucanases [10]. 
The multi-complex enzymatic cocktail known as cellulase and hemicellulase can be produced by a variety of saprophytic microorganisms. Trichoderma and Aspergillus are the genera most used to produce cellulases. Among them, one of the most productive of biomass degrading enzymes is the filamentous fungus Trichoderma reesei. It cellulolytic arsenal is composed by a mixture of endoglucanases and exoglucanases that act synergistically to break down cellulose to cellobiose. Two $\beta$-glucosidases have been identified that are implicated in hydrolyzing cellobiose to glucose. An additional protein, swollenin, has been described that disrupts crystalline cellulose structures, presumably making polysaccharides more accessible to hydrolysis. The four most abundant components of $T$. reesei cellulase together constitute more than $50 \%$ of the protein produced by the cell under inducing conditions $[9,15]$. Cellulases are essential for the biorefinery concept. In order to reduce the costs and increase production of commercial enzymes, the use of cheaper raw materials as substrate for enzyme production and focus on a product with a high stability and specific activity are mandatory. Apart from bioethanol, there are several applications to these enzymes, such as in textile, detergent, food, and in the pulp and paper industries.

\section{Enzyme production using sugarcane bagasse}

Cultivation of microorganisms in agroindustrial residues (such as bagasse) aiming the production of enzymes can be divided into two types: processes based on liquid fermentation or submerged fermentation $(\mathrm{SmF})$, and processes based on solid-state fermentation (SSF) [5]. In several SSF processes bagasse has been used as the solid substrate. In the majority of the processes bagasse has been used as the carbon (energy) source, but in some others it has been used as the solid inert support. Cellulases have been extensively studied in SSF using sugarcane bagasse. It has been reported the production of cellulases from different fungal strains [5].

Several processes have been reported for the production of enzymes using bagasse in SmF. One of the most widely studied aspects of bagasse application has been on cellulolytic enzyme production. Generally basidiomycetes have been employed for this purpose, in view of their high extracellular cellulase production. A recent example was the use of Trichoderma reesei QM-9414 for cellulase and biomass production from bagasse. Additionally, white-rot fungi were successfully used for the degradation of long-fiber bagasse. Most of the strains caused an increase in the relative concentration of residual cellulose, indicating that hemicellulose was the preferred carbon source [5].

\section{Processes for ethanol production}

Ethanol production from lignocellulosic residues is performed by fermentation of a mixture of sugars in the presence of inhibiting compounds, such as low molecular weight organic acids, furan derivatives, phenolics, and inorganic compounds released and formed during pretreatment and/or hydrolysis of the raw material. Ethanol fermentation of pentose sugars (xylose, arabinose) constitutes a challenge for efficient ethanol production from these 
residues, because only a limited number of bacteria, yeasts, and fungi can convert pentose (xylose, arabinose), as wells as other monomers released from hemicelluloses (mannose, galactose) into ethanol with a satisfactory yield and productivity [8]. Hydrolysis and fermentation processes can be designed in various configurations, being performed separately, known as separate hydrolysis and fermentation (SHF) or simultaneously, known as simultaneous saccharification and fermentation (SSF) processes.

When hydrolysis of pretreated cellulosic biomass is performed with enzymes, these biocatalysts (endoglucanase, exoglucanase, and $\beta$-glucosidase) can be strongly inhibited by hydrolysis products, such as glucose, xylose, cellobiose, and other oligosaccharides. Therefore, SSF plays an important role to circumvent enzyme inhibition by accumulation of these sugars. Moreover, because accumulation of ethanol in the fermenters does not inhibit cellulases as much as high concentrations of sugars, SSF stands out as an important strategy for increasing the overall rate of cellulose to ethanol conversion. Some inhibitors present in the liquid fraction of the pretreated lignocellulosic biomass also have a significant and negative impact on enzymatic hydrolysis. Due to the decrease in sugar inhibition during enzymatic hydrolysis in SSF, the detoxifying effect of fermentation, and the positive effect of some inhibitors present in the pretreatment hydrolysate (e.g. acetic acid) on the fermentation, SSF can be an advantageous process when compared to SHF [8]. Another important advantage is a reduction in the sensitivity to infection in SSF when compared to SHF. However, this was demonstrated by Stenberg and co-authors [12] that this is not always the case. It was observed that SSF was more sensitive to infections than SHF. A major disadvantage of SSF is the difficulty in recycling and reusing the yeast since it will be mixed with the lignin residue and recalcitrant cellulose.

In SHF, hydrolysis of cellulosic biomass and the fermentation step are carried out in different units [8], and the solid fraction of pretreated lignocellulosic material undergoes hydrolysis (saccharification) in a separate tank by addition of acids/alkali or enzymes. Once hydrolysis is completed, the resulting cellulose hydrolysate is fermented and the sugars converted to ethanol. S. cerevisiae is the most employed microorganism for fermenting the hydrolysates of lignocellulosic biomass. This yeast ferments the hexoses contained in the hydrolysate but not the pentoses. Several strategies (screening biodiversity, metabolic and evolutionary engineering of microorganisms) have been attempted to overcome this metabolic limitation.

One of the main features of SHF process is that each step can be performed at its optimal operating conditions (especially temperature and $\mathrm{pH}$ ) as opposed to SSF [7].Therefore, in SHF each step can be carried out under optimal conditions, i.e. enzymatic hydrolysis at 45$50^{\circ} \mathrm{C}$ and fermentation at $30-32^{\circ} \mathrm{C}$. Additionally, it is possible to run fermentation in continuous mode with cell recycling. The major drawback of SHF, as mentioned before, is that the sugars released during hydrolysis might inhibit the enzymes. It must be stressed out that ethanol produced can also act as an inhibitor in hydrolysis but not as strongly as the sugars. A second advantage of SSF over SHF is the process integration obtained when hydrolysis and fermentation are performed in one single reactor, which reduces the number of reactors needed. 
Experimental data from ethanol output using sugarcane bagasse as the substrate is being released in the literature. Vásquez et al. (2007) [13] described a process in which $30 \mathrm{~g} / \mathrm{L}$ of ethanol was produced in $10 \mathrm{~h}$ fermentation by S. cerevisiae (baker's yeast) at an initial cell concentration of $4 \mathrm{~g} / \mathrm{L}$ (dry weight basis) using non-supplemented bagasse hydrolysate at $37^{\circ} \mathrm{C}$. The hydrolysate was obtained by a combination of acid/alkali pre-treatment, followed by enzymatic hydrolysis. Krishnan et al. (2010) [14] reported 34 and $36 \mathrm{~g} / \mathrm{L}$ of ethanol on AFEX-treated bagasse and cane leaf residue, respectively, using recombinant $\mathrm{S}$. cerevisiae and $6 \%(\mathrm{w} / \mathrm{w})$ glucan loading during enzymatic hydrolysis. Overall the whole process produced around $20 \mathrm{~kg}$ of ethanol per $100 \mathrm{~kg}$ of each bagasse or cane leaf, and was performed during $250 \mathrm{~h}$ including pretreatment, hydrolysis and fermentation. According to the authors this is the first complete mass balance on bagasse and cane leaf.

In view of the growing concern over climate change and energy supply, biofuels have received positive support from the public opinion. However, growing concern over first generation biofuels in terms of their impact on food prices and land usage has led to an increasing bad reputation towards biofuels lately. The struggle of 'land vs fuel' will be driven by the predicted 10 times increase in biofuels until 2050. The result is that biofuels are starting to generate resistance, particularly in poor countries, and from a number of activist non-governmental organizations with environmental agendas. This is highly unfortunate as it is clear that liquid biofuels hold the potential to provide a more sustainable source of energy for the transportation sector, if produced sensibly. Since replacement of fossil fuels will take place soon, a way to avoid these negative effects from first generation biofuels (mainly produced from potential food sources) is to make lignocellulosic derived fuels available within the shortest possible time. It is known that this process involves an unprecedented challenge, as the technology to produce these replacement fuels is still being developed [1]. Fuels derived from cellulosic biomass are essential in order to overcome our excessive dependence on petroleum for liquid fuels and also address the build-up of greenhouse gases that cause global climate change [2].

\section{Author details}

T.P. Basso, T.O. Basso, C.R. Gallo and L.C. Basso

University of São Paulo, "Luiz de Queiroz" College of Agriculture, Brazil

\section{References}

[1] Gomez LD, Steele-King CG, McQueen-Mason SJ. Sustainable liquid biofuels from biomass: the writing's on the walls. New Phytologist 2008;178:473-485.

[2] Yang B, Wyman CE. Pretreatment: the key to unlocking low-cost cellulosic ethanol. Biofuels, Bioproducts and Biorefining 2008;2:26-40.

[3] Jagger A. Brazil invests in second-generation biofuels. Biofuels, Bioproducts and Biorefining 2009;3:8-10. 
[4] Galbe M, Zacchi G. A review of the production of ethanol from softwood. Applied of Microbiology and Biotechnology 2002;59:618-628.

[5] Pandey A, Socol CR, Nigam P, Soccol VT. Biotechnological potential of agro-industrial residues. I: sugarcane bagasse 2000;74:69-80.

[6] Mielenz JR. Ethanol production from biomass: technology and commercializantion status. Current Opinion in Microbiology 2001;4:324-329.

[7] Cardona CA, Quintero JA, Paz IC. Production of bioethanol from sugarcane bagasse: Status and perspectives. Bioresource and Technology 2010;101:4754-4766.

[8] Kumar S, Singh, SP, Mishra IM, Adhikari DK. Recent advances in production of bioethanol from lignocellulosic biomass. Chemistry Engineering Technology 2009;32(4)517-526. Applied Biochemistry and Biotechnology 2001;91:5-21

[9] Takashima S, Nakamura A, Hidaka M, Masaki H, Uozumi T. Molecular cloning and expression of the novel fungal $\beta$-glucosidase genes from Humicola grisea and Trichoderma reesei. Journal of Biochemistry (Tokyo) 1999;125:728-736.

[10] Zhang YHP, Himmel ME, Mielenz JR. Outlook for cellulase improvement: Screening and selection strategies. Biotechnology Advances 2006;24;452-481.

[11] Chen H, Jin S. Effect of ethanol and yeast on cellulose activity and hydrolysis of crystalline cellulose. Enzyme and Microbial Technology 2006;39:1430-1432.

[12] Stenberg K, Galbe M, Zacchi G. The influence of lactic acid formation on the simultaneous saccharification and fermentation (SSF) of softwood to ethanol. Enzyme Microbiology Technology 2000;26:71-79.

[13] Vasquez PM, Silva JNC, Souza Jr MB, Pereira Jr N. Enzymatic hydrolysis optimization to ethanol production by simultaneous saccharification and fermentation. Applied Biochemistry and Biotechnology 2007; 136-149:141-153.

[14] Krishnan C, Sousa Lda C, Jin M, Chang L, Dale BE, Balan V. Alkali-based AFEX pretreatment for the conversion of sugarcane bagasse and cane leaf residues to ethanol. Biotechnology and Bioengineering 2010; 107:441-50.

[15] Foreman PK, Brown D, Dankmeyer L, Dean R, Diener S, Dunn-Coleman NS, Goedegebuur F, Houfek TD, England GJ, Kelley AS, Meerman HJ, Mitchell T, Mitchinson C, Olivares HA, Teunissen PJM, Yao J, Ward M. Transcriptional regulation of biomass-dedrading enzymes in the filamentous fungis Trichoderma reesei. The Journal of Biological Chemistry 2003;278(34)31988-31997. 